\title{
Migration and Evaporation Characteristics of Kerosene Droplet in Supercritical Environment
}

\author{
Hailong $\mathrm{Wu}^{1}$, Wansheng Nie ${ }^{1}$, Zhi Zheng ${ }^{1}$ and Yu Liu ${ }^{1}$ \\ ${ }^{1}$ Space Engineering University, 101416Beijing, China
}

\begin{abstract}
A one-dimensional full transient droplet evaporation model was established under consideration of factors such as high pressure vapour-liquid equilibrium, high-pressure physical property corrections, gas phase dissolution, and shift of interface. The finite volume method was used for discretization to study the migration and evaporation characteristics of the surrogate fuel for kerosene, which was constituting of (mass fraction) $80 \% \mathrm{n}$-decane and $20 \% 1,2,4$-trimethylbenzene, under supercritical conditions. The results show that, under supercritical conditions, the higher the temperature and the pressure, the easier and the sooner the supercritical migration occurs. Before the supercritical migration occurring, there was obvious boundary between the gas and liquid phases. The mass fraction of component was discontinuous, and the gradient of temperature near the interface was large. After the supercritical migration occurring, the surface of the droplet disappeared, there was no obvious boundary between the gas and liquid phases, and the distribution of the components mass fraction and temperature were continuously. With the increase of the initial temperature of the droplet, the time of the supercritical migration was greatly advanced, the rise rate of the droplet surface temperature increased, and the phenomenon of endothermic expansion no longer appeared.
\end{abstract}

\section{Introduction}

With the rapid development of the aerospace industry around the world, people need to send more and more things into space for various scientific experiments, planetary exploration, and the establishment of space stations. Therefore, rocket engines with large thrust and high efficiency are urgently needed.

At present, the heavy-lift rocket engine is mainly on liquid oxygen/kerosene rocket engines. In order to improve the performance of the liquid oxygen/kerosene rocket engine, people continuously increase the pressure of the engine combustion chamber to improve the combustion efficiency. However, with the increase of the pressure, the working environment of the combustion chamber is completely supercritical. The related theories of injection, atomization, evaporation, mixing, and combustion in the past under low pressure and subcritical conditions have not been applied, especially, the process of evaporation and combustion ${ }^{[1]}$. Therefore, it is necessary to establish the theory of droplet evaporation and combustion under supercritical conditions.

The experimental research of kerosene droplet evaporation under supercritical environment is mainly on hanging-droplet method. GHASSEMI $\mathrm{H}^{[2]}$ studied the evaporation process of kerosene droplet in the pressure range of 0.1 to $3 \mathrm{MPa}$ and the temperature range of 500 to $1000{ }^{\circ} \mathrm{C}$ using the hanging-droplet method. It was found that with the increase of pressure, the evaporation rate increases first and then decreases at high temperture.
At low temperatures, when ambient pressure increased, evaporation rates monotonically increase. Jin Le et al. ${ }^{[3]}$ studied the effect of the droplet initial temperature on the supercritical evaporation of RP-3 aviation kerosene by experiments, founding that the higher the initial temperature, the larger the droplet diameter when the droplet appeared supercritical scattering spots. In terms of numerical simulation, the single-component surrogate fuel for kerosene is mainly used for studying. Li Yunqing et al. ${ }^{[4]}$ studied the process and conditions of the surface migration in the supercritical evaporation process, based on the actual gas state equation and high-pressure vapourliquid equilibrium. Li Pengfei et al. ${ }^{[5]}$ studied the variation of gas solubility, evaporation rate and droplet life under subcritical and supercritical conditions, which was based on OpenFOAM and using real fluid model and state equation method to calculate the multi-component vapour-liquid equilibrium under high-pressure. B. Balaji ${ }^{[6]}$ studied how the temperature and pressure affect droplet survival time of $n$-dodecane droplet in the subcritical and supercritical environment. There are also some multi-component supercritical evaporation models, such as Zheng $\mathrm{Li}^{[7]}$ established a multi-component evaporation model under high-temperature and highpressure, and studied of the evaporation process of dimethyl ether and liquefied petroleum gas mixture, but the supercritical model is very simple.

A one-dimensional full transient droplet evaporation model has been established and a simulation program, which can be both used for single-component and 
bicomponent droplet evaporation, was written using Fortran language. And the effectiveness of the program was verified. In the meantime, a bicomponent surrogate fuel for kerosene was found, and the transcritical characteristics of the kerosene droplet and the effect of initial droplet temperature on the transcritical characteristics of the kerosene droplet under the supercritical environment was studied by simulation.

\section{Modeling}

\subsection{Basic assumptions}

In an actual liquid rocket engine, the shape of droplet formed by atomization is not completely spherical, and the environment around the droplet are relatively complicated. So the droplet model under actual conditions is simplified for the convenience of the simulation:

(1) Ignore the airflow velocity and pressure fluctuations around the droplet.

(2) Ignore the effects of the gravity and natural convection.

(3) Ignore the shape change of droplet.

(4) Ignore Dufour and Soret Effect of Gas Phase.

(5) Ignore the effects of thermal radiation.

A one-dimensional full transient droplet evaporation model was established based on the above basic assumptions.

\subsection{Governing equations}

The governing equations of the liquid and gas phase are basically in the same form, and after the entering of the supercritical evaporation stage, the liquid phase and the gas phase no longer separate. Therefore, the unified governing equations were established for the liquid phase and the gas phase.

$$
\begin{gathered}
\frac{\partial \rho}{\partial t}+\frac{1}{r^{2}} \frac{\partial}{\partial r}\left(r^{2} \rho v\right)=0 \\
\frac{\partial\left(\rho Y_{i}\right)}{\partial t}+\frac{1}{r^{2}} \frac{\partial\left(r^{2} \rho v Y_{i}\right)}{\partial r}=\frac{1}{r^{2}} \frac{\partial}{\partial r}\left(r^{2} \rho D \frac{\partial Y_{i}}{\partial r}\right) \\
\frac{\partial\left(\rho C_{p} T\right)}{\partial t}+\frac{1}{r^{2}} \frac{\partial\left(r^{2} \rho v C_{p} T\right)}{\partial r}=\frac{1}{r^{2}} \frac{\partial}{\partial r}\left(r^{2} \lambda \frac{\partial T}{\partial r}\right)
\end{gathered}
$$

In the formula, $r$ represents the distance from a point to the center of the droplet in the physical plane $(\mathrm{m}) ; t$ represents the time(s); $Y_{\mathrm{i}}$ represents the mass fraction of the components; $\rho$ represents the $\operatorname{density}\left(\mathrm{kg} \bullet \mathrm{m}^{-3}\right)($ gas phase: $\rho_{\mathrm{g}}$, liquid phase: $\left.\rho_{1}\right) ; T$ represents the temperature $(\mathrm{K})\left(\right.$ gas phase: $T_{\mathrm{g}}$, liquid phase: $\left.T_{1}\right) ; v$ represents the velocity $\left(\mathrm{m} \bullet \mathrm{s}^{-1}\right)\left(\right.$ gas phase: $v_{\mathrm{g}}$, liquid phase: $\left.v_{1}\right) ; D$ represents the self-diffusion coefficient $\left(\mathrm{m}^{2} \cdot \mathrm{s}^{-1}\right)$ (gas phase: $D_{\mathrm{g}}$, liquid phase: $\left.D_{\mathrm{l}}\right) ; C_{\mathrm{p}}$ represents the specific heat at constant pressure $\left(J \cdot \mathrm{kg}^{-1} \cdot K^{-1}\right)$ (gas phase: $C_{\mathrm{pg}}$, liquid phase: $\left.C_{\mathrm{pl}}\right) ; \lambda$ represents the coefficient of heat conduction $\left(W \cdot m^{-1} \cdot K^{-1}\right)$ (gas phase: $\lambda_{\mathrm{g}}$, liquid phase: $\left.\lambda_{1}\right)$.

\subsection{Initial and boundary conditions}

\subsubsection{Initial conditions}

At the time of $t=0$,

$$
T_{1}=T_{10}, T_{\mathrm{g}}=T_{\infty}, P_{\mathrm{g}}=P_{1}=P_{\infty}
$$

$T_{10}$ represents the initial temperature of the droplet, $T_{\infty}$ and $P_{\infty}$ represent the temperature and pressure at the boundary of infinity. At the initial conditions, there are only fuel components inside the droplet, and only ambient gas components in the gas phase.

\subsubsection{Subcritical boundary conditions}

In the infinity of gas phase:

$T=T_{\infty} 、 Y_{\mathrm{i}}=0$ or 1 (it is 0 when the i represents the fuel component and it is 1 when the $\mathrm{i}$ represents the nitrogen).

At the center of the droplet:

$$
\frac{\partial T}{\partial r}=0, \frac{\partial Y_{\mathrm{i}}}{\partial r}=0, \frac{\partial v}{\partial r}=0
$$

At the surface of the droplet:

$$
T_{g, s}=T_{l, s}=T S, \phi_{i}^{l} X_{i}^{l}=\phi_{i}^{g} X_{i}^{g} .
$$

Of which, $T_{\mathrm{g}, \mathrm{s}}$ and $T_{1, \mathrm{~s}}$ are the gas-phase and liquidphase temperatures of the droplet surface, respectively. $T S$ is temperature of the droplet surface. $X_{i}^{g}$ and $X_{i}^{g}$ are respectively the gas-phase and liquid-phase fugacity coefficient of the droplet surface, which were Obtained by the Peng-Robinson equation of state ${ }^{[8]}$.

\subsubsection{Supercritical boundary conditions}

When the temperature and pressure at the gas-phase of droplet surface exceed the critical value of the mixture, it is considered that the supercritical state has reached. At this time, the surface tension of the droplet is reduced to zero, the surface of the droplet disappears, and the thermal properties between the gas and the liquid phase tend to continuous ${ }^{[9]}$. Therefore, when the surface of the droplet reaches a supercritical state, the gas-liquid phase is no longer distinguished, and only the boundary conditions at the centre and the surface of the droplet are defined, and its definition is the same as the subcritical state.

\subsection{Numerical computation method}

The surface of the droplet changes with the evaporation process, if the control equation is directly discretized and solved, it is necessary to repartition the calculation grid at each time step. In order to avoid it, using the formulas (7) and (8) to convert the coordinate ${ }^{[8]}$ from the physical 
plane to the calculation plane to make the droplet surface always fixed at the position where the calculation coordinate is 1 .

Spatial position transformation of two coordinates:

$$
\zeta=r / R_{\mathrm{S}}
$$

Velocity transformation of two coordinates:

$$
w=v-\zeta \dot{R}_{\mathrm{S}}
$$

Of which, $\zeta$ represents the distance from a point to the center of the droplet in the calculation plane, $R_{\mathrm{s}}$ represents the radius of the $\operatorname{droplet}(\mathrm{m}), \quad \dot{R}_{\mathrm{S}}$ represents the change rate of the droplet radius.

Since the flow field parameters near the surface of the droplet varies greatly, the grid is divided into nonuniform grids near the surface of the droplet to make the grid refinement, thereby improving the calculation accuracy of the flow field near the droplet surface.

The finite volume method is used for the discrete method of the governing equations with coordinate conversion, and the control volume boundary is taken as the centre of the adjacent grid node ${ }^{[10]}$.

\section{Model verification}

In order to test the effectiveness of the calculation model and the program, the evaporation process of n-heptane droplet with a diameter of $50 \mu \mathrm{m}$ under different pressures and temperatures in a nitrogen atmosphere was calculated, and the results were compared with the results of the literature [9]. As shown in Figure 1.

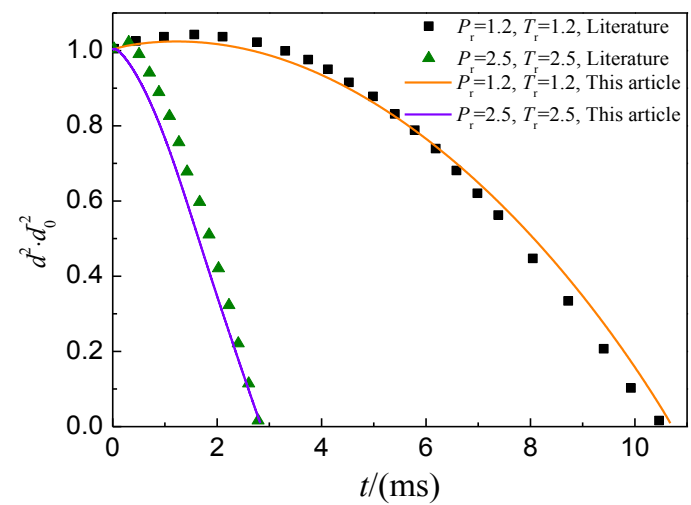

Figure 1. Simulation result comparison and verification

In the figure $1, P r$ represents the ratio of ambient pressure to $\mathrm{n}$-heptane critical pressure. $T_{\mathrm{r}}$ represents the ratio of ambient temperature to n-heptane critical temperature. $d$ and $d_{0}$ represents the diameter and initial diameter of the droplet, respectively. It can be seen that the calculation results in this paper is in good agreement with the calculations in the literature under the conditions not only of lower temperature and pressure but also of higher temperature and pressure. The correctness of the model has been verified.

\section{Calculation results and analysis}

\subsection{The selection of surrogate fuel for kerosene}

At present, the model number of aviation kerosene mainly used in our country is RP-3. The composition of RP-3 aviation kerosene was analysed by gas chromatography-mass spectrometry in literature [11]. The results show that RP-3 is composed of $42.330 \%$ paraffins, $21.348 \%$ cycloparaffins, $24.045 \%$ benzenes, $8.184 \%$ naphthalenes, and $4.093 \%$ others. RP-3 aviation kerosene is a complex mixture. Now, it is impossible to directly perform the simulation calculation of evaporation process of the complex mixture droplet under the supercritical environment. This article uses the bicomponent surrogate fuel for RP-3 aviation kerosene proposed in [12] and [13]: (mass fraction) $80 \%$ n-decane and $20 \% \quad 1,2,4$-trimethylbenzene. Its main thermodynamic properties are shown in Table 1 . There are several main reasons for using this solution:

(1) The simulation of the bicomponent surrogate fuel is easier to realize.

(2) Compared to single-component surrogate fuel for kerosene, bicomponent surrogate fuel for kerosene can simulate the mixture characteristics of kerosene.

(3) From analyzing the components of kerosene, it can be seen that alkane and benzene series are the main components. Selecting n-decane and 1,2,4trimethylbenzene can be a good substitute for the physical and chemical properties of alkanes and benzenes, and laying a good foundation for the subsequent simulation of kerosene droplet combustion.

Table 1. The components and thermodynamic properties of the bicomponent surrogate fuel for kerosene.

\begin{tabular}{|c|c|c|c|c|}
\hline $\begin{array}{c}\text { Compo } \\
\text { nent }\end{array}$ & $\begin{array}{c}\text { mass } \\
\text { fraction } \\
(\%)\end{array}$ & $\begin{array}{c}\text { Critical } \\
\text { pressure } \\
\text { (atm) }\end{array}$ & $\begin{array}{c}\text { Critical } \\
\text { temperature } \\
(\mathrm{K})\end{array}$ & $\begin{array}{c}\text { Boiling } \\
\text { point } \\
(\mathrm{K})\end{array}$ \\
\hline $\begin{array}{c}\mathrm{n}- \\
\text { decane }\end{array}$ & 80 & 20.8 & 617.7 & 447.3 \\
\hline $\begin{array}{c}1,2,4- \\
\text { trimeth } \\
\text { ylbenze } \\
\text { ne }\end{array}$ & 20 & 31.9 & 649.1 & 442.5 \\
\hline
\end{tabular}

\subsection{The evaporation of droplet in a supercritical environment}

In this paper, a bicomponent surrogate fuel, which was composed of n-decane and 1,2,4-trimethylbenzene, for kerosene droplet is used in the calculation. The initial diameter of the droplet $d_{0}$ is $100 \mu \mathrm{m}$, and the initial temperature of the droplet $T_{10}$ is $300 \mathrm{~K}$. The surrounding gas is nitrogen.

In the figure 2 , it shows the variation of the relative diameter square of the droplet and the surface temperature of the droplet under three working conditions $P=80 \mathrm{~atm} T=1200 \mathrm{~K}, P=100 \mathrm{~atm} T=1400 \mathrm{~K}$ and $P=120 \mathrm{~atm}$ $T=1600 \mathrm{~K}$. In the initial stage of droplet evaporation, the surface temperature of the droplet rapidly rises, and the droplet diameter increases. This is mainly because of the large temperature differential between the droplet initial temperature and the ambient temperature. The droplet 
rapidly absorbs heat and expands, but at this time almost all of the absorbed heat is used to increase the temperature of the droplet, causing the evaporation rate of the droplet to be close to zero, the droplet to be thermally expanded, and the diameter of the droplet to increase. As the temperature differential between the droplet surface and the ambient temperature decreases, the droplet enters the rapid evaporation stage. Although the surface temperature of the droplet increases, the increase rate is little, and the droplet diameter rapidly decreases until the droplet evaporation finishes or the super critical migration occurs.

With the increase of the ambient pressure and temperature, the temperature rising rate of the droplet surface in the initial stage of evaporation increases, but the maximum temperature that can be achieved is almost the same. The decreasing rate of the square of the droplet diameter is also increasing. At the initial stage of evaporation, the phenomenon of heat absorption and expansion of droplet becomes less and less obvious. At the same time, the higher the pressure and temperature are, the earlier the supercritical migrate of the droplet occurs.

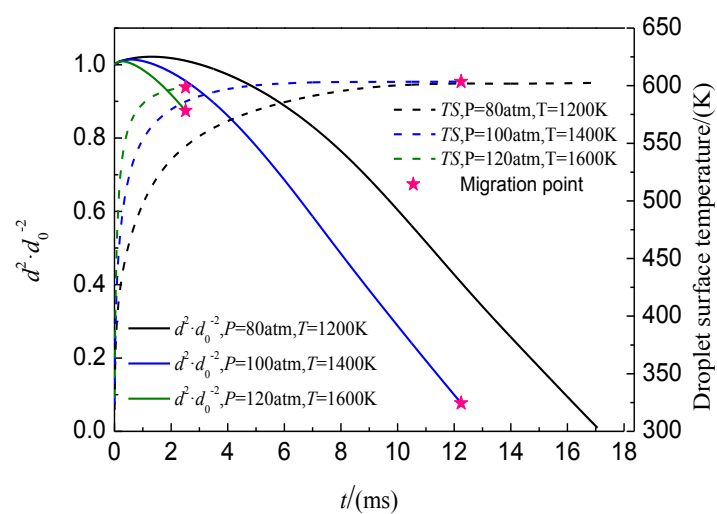

Figure 2. Variation of droplet relative diameter square and droplet surface temperature

\subsection{The evaporation characteristics of droplet before and after supercritical migration}

In the figure 3 , it shows the distribution of the mass fraction and temperature along the radius of each component of the droplet before and after the supercritical migration at $P=120 \mathrm{~atm}$ and $T=1600 \mathrm{~K}$. Under the condition, the droplet life is $13.61 \mathrm{~ms}$, and the migration time is $2.51 \mathrm{~ms}$. The time of $1.25 \mathrm{~ms}$ and 7.51 $\mathrm{ms}$ are selected, which is the middle moment of two stages before and after the supercritical migration.

It can be seen that the mass fraction of $n$-decane and 1,2,4-trimethylbenzene inside the droplet changes very little before the occurrence of supercritical migration, which is basically the initial value, and the mass fraction of nitrogen is almost zero. In the vicinity of the droplet surface, there is an obvious discontinuity, that is to say the mass fraction of the components is not continuous at the interface. At the same time, the liquid phase temperature is also low, high temperature only occurs near the droplet surface. Although the temperature at the gas-liquid interface is continuous, the temperature gradient is large.

After the occurring of the supercritical migration, the mass fraction of $\mathrm{n}$-decane and 1,2,4-trimethylbenzene in the original liquid phase of the droplet decreases significantly, and the mass fraction of nitrogen increases. Along the radius $r$, the mass fraction of the components is continuously distributed and there is no interruption. At the same time, the temperature inside the original phase of the droplet increased and the temperature gradient decreased significantly.

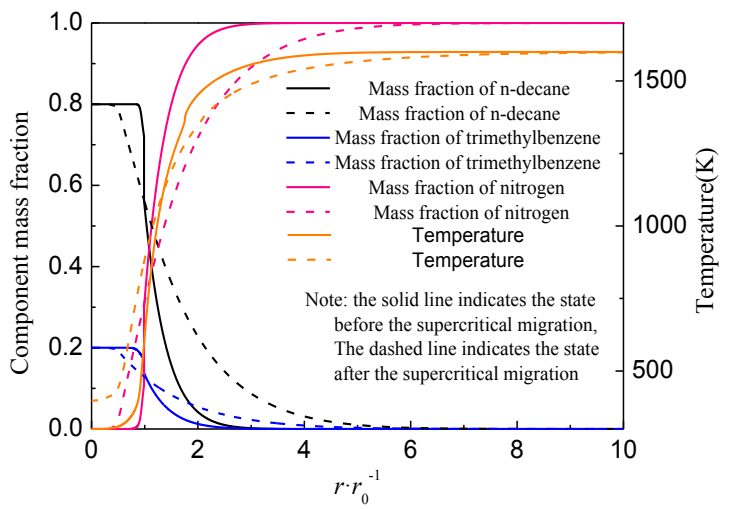

Figure 3. The distribution of component mass fraction and temperature

\subsection{The effect of initial droplet temperature on the supercritical migration}

Figure 4 and 5 are the time-dependent curves of the droplet relative diameter square and the droplet surface temperature under the initial conditions of $P=100 \mathrm{~atm}$ $T=1400 \mathrm{~K}$, respectively. It can be seen that under the same environmental conditions, the initial temperature of the droplet has a great influence on the evaporation characteristics of the droplet, especially the supercritical migration characteristics. As the initial temperature of the droplet increases, the point of supercritical migration of the droplet occurs more and more earlier, and which even occurs immediately at the beginning of evaporation.

In figure 4, as the initial temperature of the droplet increases, the endothermic expansion phase of the droplet evaporation process becomes more and more unapparent. When the droplet initial temperature reaches $450 \mathrm{~K}$, there is no endothermic expansion phase. At the same time, it can be seen from figure 5 that with the increase of the initial droplet temperature, the rise rate of droplet surface temperature significantly increases although the initial temperature differential between the gas and liquid phases decreases. The above phenomenon occurs mainly because the initial temperature of the droplet becomes closer to the boiling point of the mixture, as the initial temperature of the droplet increases, the more easily the gasification occurs.

Therefore, it can be seen that in the actual engine working process, the combustion efficiency can be improved by increasing the initial temperature of the injected fuel to accelerate the evaporation rate of the atomized droplet. Especially in the ignition phase, 
accelerating the evaporation rate can greatly reduce the ignition delay.

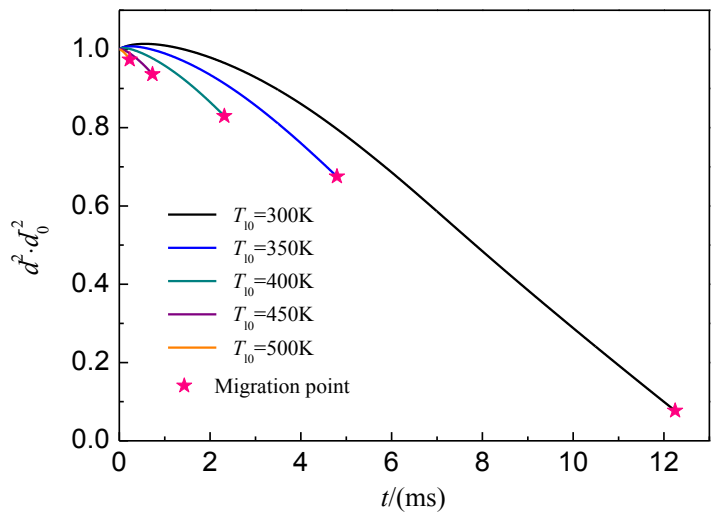

Figure 4. Variation of droplet relative diameter square

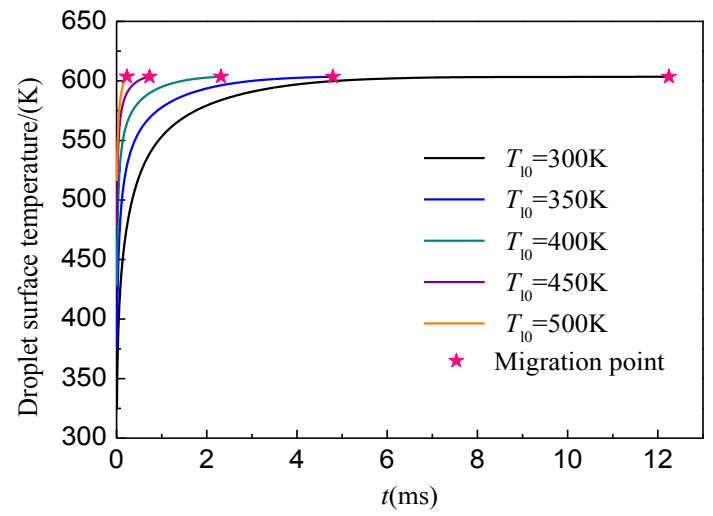

Figure 5. Variation of droplet surface temperature

\section{Conclusion}

The paper first establishes a simulation calculation program for the droplet non-steady-state evaporation model in supercritical environment and verifies the validity of the calculation program, then searched for a bicomponent surrogate fuel for RP-3 aviation kerosene. Finally, the evaporation characteristics, supercritical transport characteristics and effects of initial droplet temperature on evaporation and migration characteristics in supercritical environment were studied by simulation calculations. The results not only lay the foundation for the simulation research of kerosene droplet combustion in the supercritical environment, but also provide some reference for explaining the mechanism of ignition and unstable combustion in liquid oxygen/kerosene rocket engines. The main conclusions are as follows:
(1) Under supercritical conditions, as the ambient temperature and pressure increase, the droplet are more prone to occurring supercritical migration and the earlier the supercritical migration occurs.

(2) Before the occurrence of supercritical migration, there were obvious boundaries between the gas and liquid phases, the distribution of mass fraction of each component was discontinuous and the gradient of temperature near the interface was large. After the supercritical migration occurs, the droplet surface disappears, there is no obvious boundary between the gas and liquid phases, and the mass fraction and temperature of each component are continuously distributed.

(3) With the increase of the initial temperature of the droplet, the supercritical fluid migration point advances significantly. The rise rate of the droplet surface temperature increases, and the droplet no longer displays the phenomenon of thermal expansion.

\section{References}

1. F. C. Zhuang, National University of Defence Technology Press. M(1995)

2. H. GHASSEMI, S. W. BAEK, Q. S. KHAN, Combustion Science and Technology. J. 178. 16691684(2006)

3. L. Jin, W. Fan, Z. Zhou, et al. Journal of Combustion Science and Technology. J. 22. 269-275(2016)

4. Y. Q. Li, H. N. Wang, W. Chen, Journal of Combustion Science and Technology. J. 16. 287-294 (2010)

5. P. F.Li, F. P. Lei, K. Wang, et al. Acta Aeronautica et Astronautica Sinica. J. 39. 121764(2018)

6. B. Balaji, Vasudevan Raghavan, K. Ramamurthi, et al. Physics of Fluids. J. 23.063601(2011)

7. L. Zheng, Wuhan University of Technology. D(2014)

8. R. Ruszalo, The University of Ottawa. D(1990)

9. P. He, School of Transportation Science and Engineering Beihang University. D(2010)

10. F. J. Wang, Tsinghua Universit Press. M(2004)

11. Z. Zhou, W. Fan, L. Jin, et al. Journal of Propulsion Technology. J. 37. 1422-1430(2016)

12. H. R. Wang, Gas Turbine Experiment and Research. J. 28. 19-23(2015)

13. S. Honnet, K. Seshadri, U. Niemann, et al. Proceedings of the Combustion Institute. J. 32. 485 492(2009) 